\title{
miR-488 negatively regulates osteogenic differentiation of bone marrow mesenchymal stem cells induced by psoralen by targeting Runx2
}

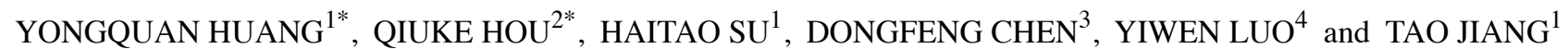 \\ ${ }^{1}$ Department of Orthopaedics, The Second Affiliated Hospital of Guangzhou University of Chinese Medicine, Guangzhou, \\ Guangdong 510006; ${ }^{2}$ Department of Gastroenterology, The First Affiliated Hospital of Guangzhou University of \\ Chinese Medicine, Guangzhou, Guangdong 510405; ${ }^{3}$ Department of Anatomy, Guangzhou University of Chinese Medicine, \\ Guangzhou, Guangdong 510006; ${ }^{4}$ Department of Trauma, The Third Affiliated Hospital of Guangzhou \\ University of Chinese Medicine, Guangzhou, Guangdong 510360, P.R. China
}

Received September 9, 2018; Accepted May 14, 2019

DOI: $10.3892 / \mathrm{mmr} .2019 .10613$

\begin{abstract}
It has been previously reported that psoralen, one of the active ingredients in Psoralea corylifolia, could induce osteogenic differentiation of bone marrow mesenchymal stem cells (BMSCs), suggesting its potential to treat osteoporosis. Additionally, runt-related transcription factor 2 (Runx2) is a transcription factor that plays vital roles in BMSC osteogenic differentiation. However, whether and how microRNAs (miRNAs/miRs) modulate osteogenic differentiation induced by psoralen have not yet been examined, to the best of the authors' knowledge. The present study aimed to identify the miRNA target genes that regulate osteogenic differentiation of BMSCs induced by psoralen. A Cell Counting Kit- 8 assay and alizarin red staining were used to detect the viability and osteogenic differentiation of BMSCs, respectively, under treatment with psoralen. miRNA microarray analysis was performed to identify the differentially expressed miRNAs under treatment with psoralen. A bioinformatics analysis and a luciferase reporter assay were conducted to identify the targets of miR-488. Finally, the mechanisms of miR-488
\end{abstract}

Correspondence to: Mr. Tao Jiang, Department of Orthopaedics, The Second Affiliated Hospital of Guangzhou University of Chinese Medicine, 55 Inner Ring West Road, Guangzhou Higher Education Mega Center, Guangzhou, Guangdong 510006, P.R. China E-mail: iq_591@163.com

Mr. Yiwen Luo, Department of Trauma, The Third Affiliated Hospital of Guangzhou University of Chinese Medicine, 261 Longxi Avenue, Liwan, Guangzhou, Guangdong 510360, P.R. China

E-mail: gzzyydxlyw@126.com

*Contributed equally

Key words: bone marrow mesenchymal stem cells, psoralen, microRNA-488, runt-related transcription factor 2, osteogenic differentiation in psoralen-induced BMSC osteogenic differentiation were investigated using overexpression or inhibition methods in vitro. Cell viability was elevated and osteogenic differentiation of BMSCs was improved under treatment with psoralen. miRNA microarray analysis and further validation by reverse transcription-quantitative PCR revealed that miR-488 was downregulated during psoralen-induced BMSC osteogenic differentiation. Bioinformatics analysis and experimental validation by a luciferase reporter assay identified Runx 2 as a potential target of miR-488. Overexpression of miR-488 by transfection with miR-488 mimics markedly inhibited the expression of Runx2, Osterix and alkaline phosphatase, whereas, the inhibition of miR-488 expression by the miR-488 inhibitor promoted their expression compared with the control. Rescue assays demonstrated that Runx 2 overexpression partially rescued the inhibitory effect of miR-488 on BMSC osteogenic differentiation. The present results suggested that miR-488 is a negative regulator of psoralen-induced BMSC osteogenic differentiation by targeting Runx2, providing a possible therapeutic target for osteoporosis.

\section{Introduction}

Osteoporosis is a systemic skeletal disease common in older women, which is characterized by low bone mass and micro-architectural deterioration of bone tissue, followed by increasing bone fragility and susceptibility to fracture (1). It is estimated that $>50 \%$ of women aged 50 years and older will sustain an osteoporotic fracture during their lifetime (2). However, at present, it is difficult to achieve satisfactory clinical efficacy for the treatment of osteoporosis due to the side effects of drugs and patient compliance $(1,3)$. Promoting osteogenic differentiation is an important strategy to enhance bone mineral density and slow the development of osteoporosis (4). Bone marrow mesenchymal stem cells (BMSCs) are mesodermal cells that can be obtained from multiple sources, including adipose tissue, periosteum and bone marrow (5). Due to their unique capacity to differentiate into osteoblasts, chondrocytes and adipocytes, BMSCs are widely used in osteoporosis 
research worldwide (6,7). Psoralea corylifolia L., a traditional Chinese herbal medicine, has a long history of clinical efficacy against conditions such as fractures, bone defects and osteoporosis (8). Psoralen, as the main flavonoid active ingredient in Psoralea corylifolia L., was demonstrated to promote BMSCs to undergo osteogenic differentiation through activation of the bone morphogenetic protein (BMP) signalling pathway (9). However, the mechanisms underlying the osteogenic differentiation effects of psoralen have not yet been fully elucidated, to the best of the authors' knowledge, and the epigenetic regulatory mechanisms required investigation.

In recent decades, it has been demonstrated that short non-coding RNAs, microRNAs (miRNAs/miRs), are involved in many biological processes, including cell survival, proliferation and differentiation, while their aberrant expression can lead to the development of diseases (10). Notably, miRNAs are also the central regulators of BMSCs in regulating osteogenic differentiation. miR-203 and miR-320 negatively regulated BMP-2-induced osteogenic differentiation by suppressing homeobox protein DLX-5 (11). miR-195 inhibited the abnormal activation of osteogenic differentiation in MC3T3-E1 cells by targeting RAF proto-oncogene serine/threonine-protein kinase (12). miR-495 inhibited new bone regeneration by targeting high mobility group at-hook 2 (13). Therefore, the regulation of osteogenic transcription factors by miRNAs was demonstrated to be an effective strategy in regulating osteogenic differentiation. Runt-related transcription factor 2 (Runx2) is a transcription factor that is indispensable for skeletal development, and controls bone formation by acting as a signalling hub and transcriptional regulator to coordinate target gene expression $(14,15)$. However, the role of miRNAs in targeting Runx2 requires clarification in the osteogenic differentiation of BMSCs.

In the present study, BMSCs treated with psoralen were used to identify differentially expressed miRNAs and their target genes via miRNA microarray and bioinformatics analysis. Using overexpression or inhibition methods in vitro, the underlying mechanisms of miRNAs in psoralen-induced BMSC osteogenic differentiation were examined, providing a potential molecular therapeutic strategy for osteoporosis.

\section{Materials and methods}

Isolation and culture of BMSCs. 20 male Sprague-Dawley rats, 3 weeks old (weighing 140 $\pm 20 \mathrm{~g}$ ), obtained from the Experimental Animal Center of Guangzhou University of Traditional Chinese Medicine (Guangzhou, China), were sacrificed by decollation and were sterilised using $75 \%$ ethanol. The marrow from the femur and tibia was mixed with complete medium (low-glucose DMEM (Gibco; Thermo Fisher Scientific, Inc.) supplemented with 10\% FBS (Gibco; Thermo Fisher Scientific, Inc.)) and was gradient centrifuged at $900 \mathrm{x} \mathrm{g}$ for $30 \mathrm{~min}$ at room temperature with Percoll at a density of $1.073 \mathrm{~g} / \mathrm{ml}$. The cells were cultivated with complete medium, with fresh media changes every 3 days, and were incubated at $37^{\circ} \mathrm{C}$ in $5 \% \mathrm{CO}_{2}$. Primary rat BMSCs were passaged 6-7 days later. As the BMSCs were obtained from rats, there were many other cells in the primary, first-passage and second-passage cells. The purity $(>95 \%)$ and cell viability were high in the third-passage BMSCs, and thus were used for subsequent experiments. All the rats received humane care in accordance with the guidelines set by the Care of Experimental Animals Committee of Guangzhou University of Chinese Medicine. Additionally, the present study was approved by the Ethics Committee of Guangzhou University of Chinese Medicine. All rats were housed in a specific-pathogen-free facility $\left(22 \pm 2^{\circ} \mathrm{C}\right.$, relative humidity $60 \pm 10 \%$ ), under a $12 / 12 \mathrm{~h}$ light/dark cycle (lights on from 6:00 am), without restriction to food and water, and fasted $12 \mathrm{~h}$ before sacrifice.

Cell Counting Kit-8 (CCK-8) assay of cell viability. Third-passage BMSCs were collected and seeded into 96-well plates at a density of $1 \times 10^{4}$ cell $/ \mathrm{ml}$ and were co-cultured with $20 \mu \mathrm{g} / \mathrm{ml}$ psoralen continuously $\left(37^{\circ} \mathrm{C}\right)$, which was purchased from The National Institute for the Control of Pharmaceutical and Biological Products. Next, $10 \mu \mathrm{lCCK}-8$ solution (Dojindo Molecular Technologies, Inc.) was added into each well after cell culture for $12,24,36,48$ and $72 \mathrm{~h}$. The cells were incubated at $37^{\circ} \mathrm{C}$ for $2 \mathrm{~h}$ in the dark. The absorbance values at a wavelength of $490 \mathrm{~nm}$ were detected using a microplate reader (Bio-Rad Laboratories, Inc.). BMSCs that were not co-cultured with psoralen were considered the control group. Each experiment was repeated three times.

Alizarin red staining (AR-S) after osteogenic differentiation. Third-passage BMSCs were seeded into 24-well plates at a density of $5 \times 10^{4}$ cell $/ \mathrm{ml}$ and then were divided into three groups: Psoralen group, positive control group and blank control group. BMSCs co-cultured with $20 \mu \mathrm{g} / \mathrm{ml}$ psoralen were considered the psoralen group. The positive control group was induced by $\alpha$-Minimum Essential Medium (Gibco; Thermo Fisher Scientific, Inc.) containing $5 \times 10^{-5} \mathrm{~mol} / 1$ isobutyl xanthine, $2 \times 10^{-4} \mathrm{~mol} / 1$ indomethacin, $1 \times 10^{-5} \mathrm{~mol} / 1$ dexamethasone and $10 \mathrm{mg} / 1$ insulin. BMSCs that were not co-cultured with psoralen or osteogenic-inducing fluid were treated as the blank control group. Medium containing psoralen or osteogenic-inducing fluid was changed every 3 days. After 2 weeks, the BMSCs were fixed with $95 \%$ ethanol $10 \mathrm{~min}$ at room temperature and then were stained with $40 \mathrm{mM}$ AR-S solution for $10 \mathrm{~min}$ at $\mathrm{pH} 4.2$ (room temperature). Subsequently, the BMSCs were treated with $10 \%$ cetylpyridinium chloride in $10 \mathrm{mM}$ sodium phosphate for $15 \mathrm{~min}$ at room temperature and then were washed with PBS for $15 \mathrm{~min}$. Calcium mineral deposition was observed using a light microscope at three different views (magnifications, x100 and 200) to compare the degree of osteogenic differentiation between the different groups.

miRNA microarray analysis. Third-passage BMSCs were seeded at a density of $1 \times 10^{5}$ cell $/ \mathrm{ml}$ in a 6 -well plate. After reaching $80-85 \%$ confluence, the BMSCs treated with $20 \mu \mathrm{g} / \mathrm{ml}$ psoralen represented the psoralen group, while the BMSCs that did not receive treatment with psoralen represented the control group. After $72 \mathrm{~h}$, the total RNA of the two groups was extracted using TRIzol ${ }^{\circledR}$ reagent (Invitrogen; Thermo Fisher Scientific, Inc.) according to the manufacturer's protocol. miRNA microarray analysis was then performed by Guangzhou RiboBio Co., Ltd. The differentially expressed miRNAs were verified by reverse transcription-quantitative PCR (RT-qPCR). 
$R T-q P C R$. RT-qPCR was performed using the Prime Script ${ }^{\mathrm{TM}}$ RT reagent kit and SYBR Premix EX Taq II kit (Invitrogen; Thermo Fisher Scientific, Inc.) according to the manufacturer's protocol. The thermocycling conditions for qPCR were as follows: Pre-denatured at $95^{\circ} \mathrm{C}$ for $10 \mathrm{~min}$, following by 40 cycles of $95^{\circ} \mathrm{C}$ denaturation for $10 \mathrm{sec}$, annealing at $60^{\circ} \mathrm{C}$ for $20 \mathrm{sec}$ and finally extension $70^{\circ} \mathrm{C}$ for $15 \mathrm{sec}$. U6 was used as the internal reference for miRNA, and GAPDH was used as the internal reference for osteogenic-specific factors, and the relative expression levels were calculated using the $2^{-\Delta \Delta \mathrm{Cq}}$ method (16). The primers were synthesized by Sangon Biotech Co., Ltd. and were as follows: Runx2 (forward: 5'-TCTTAG AACAAATTCTGCCCTTT-3'; reverse: 5'-TGCTTTGGT CTTGAAATCACA-3'); Osterix (forward: 5'-AGAGATCTG AGCTGGGTAGAGG-3'; reverse: 5'-AAGAGAGCCTGG CAAGAGG-3'); alkaline phosphatase (ALP; forward: 5'-CCA ACTCTTTTGTGCCAGAGA-3'; reverse: 5'-GGCTACATT GGTGTTGAGCTTTT-3'); GAPDH (forward: 5'-ATTTGG TCGTATTGGGCG-3'; reverse: 5'-TGGAAGATGGTGATG GGATT-3'); miR-122: (forward: 5'-GCGAAAGCATTTGCC AAGAA-3', reverse: 5'-CATCACAGACCTGTTATTGC-3'); miR-154 (forward: 5'-TAGGTTATCCGTGTTGCCTTCG-3'; reverse: 5'-AAGGCAACACGAUAACCUAUU-3'); miR-488 (forward: 5'-CGGGGCAGCUCAGUACAG-3'; reverse: 5'-CAGTGCGTGTCGTGGAGT-3'); miR-205 (forward: 5'-CCTCCCTAAATCCTCCATCC-3'; reverse: 5'-TCTAGG AAGGACAGCCTCCA-3'); U6 (forward: 5'-CTCGCTTCG GCAGCACA-3'; reverse: 5'-AACGCTTCACGAATTTGC GT-3').

Bioinformatics analysis. TargetScan (http://www.targetscan. org), PicTar (http://pictar.mdc-berlin.de) and miRanda (http://www.microrna.org) were used to analyse potential miR-488 binding sites on the Runx2 3'-untranslated region (3'UTR). The consistency of the analyses and predictions using these three websites suggests that they are reliable, and the results were further verified by in vitro experiments.

RNA oligoribonucleotide synthesis and transfection. The RNA oligoribonucleotides [miR-488 mimics (5'-GGGTCT ATTACCGTGAGAGTT), miR-488 inhibitor (TTGAGAGTG CCATTATCTGGG-3'), mimics control (5'-TACGTCCAAGG TCGGGCAGGAAGA-3'), inhibitor control (5'-UCCUCC GAACGUGUCACGUTT-3'), pcDNA 3.1-Runx2 and pcDNA 3.1-control] used in the present study were synthesized by Shanghai GenePharma Co., Ltd. Prior to transfection, BMSCs were isolated and seeded $\left(2 \times 10^{6}\right.$ cells $\left./ 1\right)$ into 6 -well plates and grown until they were $60-80 \%$ confluent. The cells were then transfected with $100 \mathrm{nM}$ miR-488 mimics, miR-488 inhibitor, mimics control, inhibitor control, pcDNA 3.1-Runx2 or pcDNA 3.1-control for $6 \mathrm{~h}$, using Lipofectamine ${ }^{\circledR} 2000$ (Invitrogen; Thermo Fisher Scientific, Inc.). The cells were then digested with $0.025 \%$ trypsin for $24 \mathrm{~h}$ and collected for further analyses, including RTq-PCR, western blotting, a luciferase reporter assay and immunocytochemistry.

Luciferase reporter assay. Third-passage BMSCs were collected following $0.25 \%$ trypsin digestion when the BMSCs reached $80 \%$ confluence. The cells were transfected with $0.2 \mu \mathrm{g}$ pLUC-Runx2 plasmid construct (Guangzhou
RiboBio Co., Ltd.) and miR-488 mimics or inhibitor for $6 \mathrm{~h}$ using Lipofectamine ${ }^{\circledR}$ 2000. The activity in each well was measured using the Dual Luciferase Reporter Assay System (Promega Corporation) to quantify the luminescent signal. Firefly luciferase was selected as the internal reference. The relative luciferase activity was calculated as the value of each group/the value of miR-control group.

Rescue assays. The open reading frame (ORF) fragments of Runx2 without the 3'UTRs were amplified by PCR as aformentioned using Taq DNA Polymerase (Beijing Solarbio Science $\&$ Technology Co., Ltd.), and then Runx 2 recombinant vectors were constructed using the pcDNA 3.1 plasmid (Shanghai GenePharma Co., Ltd.). The thermocycling conditions were as follows: Pre-denatured at $94^{\circ} \mathrm{C}$ for $5 \mathrm{~min}$, followed by 35 cycles at $94^{\circ} \mathrm{C}$ for $10 \mathrm{sec}, 60^{\circ} \mathrm{C}$ for $45 \mathrm{sec}$ and $72^{\circ} \mathrm{C}$ for $1 \mathrm{~min}$. Third-passage BMSCs were seeded at a density of $2 \times 10^{4}$ cell $/ \mathrm{cm}^{2}$ into 6 -well plates and then were transfected with pcDNA 3.1-Runx2 or miR-488 mimics or co-transfected with both constructs. Cells transfected without any plasmid were treated as the control group. RT-qPCR, western blotting, AR-S staining and immunocytochemistry were then used to detect the degree of osteogenic differentiation.

Western blotting. The protein was extracted from BMSCs by radioimmunoprecipitation assay lysis buffer $(50 \mathrm{mM}$ Tris- $\mathrm{HCl}$ (pH 7.4), $150 \mathrm{mM} \mathrm{NaCl}, 1 \% \mathrm{NP}-40,0.1 \%$ SDS). The supernatant was obtained by centrifugation at $12,000 \times \mathrm{g}$ at $4^{\circ} \mathrm{C}$ for $20 \mathrm{~min}$, and the concentration of protein was determined by a bicinchoninic acid assay (Pierce; Thermo Fisher Scientific, Inc.). Then $20 \mu \mathrm{g}$ protein was added to $10 \%$ SDS-PAGE for electrophoresis at a constant electric current of $25 \mathrm{~mA} / \mathrm{gel}$ to the bottom of separation gel. The proteins were then transferred onto a PVDF membrane through electrophoresis at normal temperature with constant voltage of $80 \mathrm{~V}$ for $100 \mathrm{~min}$, and the membrane was blocked with 5\% non-fat milk in PBS with Tween-20 solution at $4{ }^{\circ} \mathrm{C}$ overnight. The blots were probed with primary antibodies against Runx2 (Abcam, ab114133, 1:2,000), Osterix (Abcam, ab209484, 1:2,000), ALP (Abcam, ab83259, 1:2,000) and $\beta$-actin (Abcam, ab8227, 1:2,000) at room temperature for $2 \mathrm{~h}$, followed by incubation with relevant secondary antibodies (Abcam, ab97051, ab150118, 1:2,000) labelled with horseradish peroxidase at room temperature for $1 \mathrm{~h}$ and washing with TBS with $0.1 \%$ Tween-20. The protein expression was detected using a SuperSignal West Femto Maximum Sensitivity Substrate kit (Roche Applied Science). The relative value of the target protein was calculated by comparing with the corresponding internal reference. Densitometry was conducted using ImageJ (v1.8.0; National Institutes of Health).

Immunocytochemistry. After transfection, the BMSCs were fixed with $4 \%$ polyoxymethylene for $20 \mathrm{~min}$ at room temperature, permeabilized with $0.25 \%$ Triton $\mathrm{X}-100$ (Sigma-Aldrich; Merck KGaA), and blocked with $0.1 \%$ bovine serum albumin (Roche Diagnostics) at room temperature for 30 min. Subsequently, the BMSCs were incubated overnight at $4{ }^{\circ} \mathrm{C}$ with the following primary antibodies: Runx2 (Abcam, ab114133, 1:500), Osterix (Abcam, ab209484, 1:500), ALP (Abcam, ab83259, 1:500). After washing three times with PBS, 
A
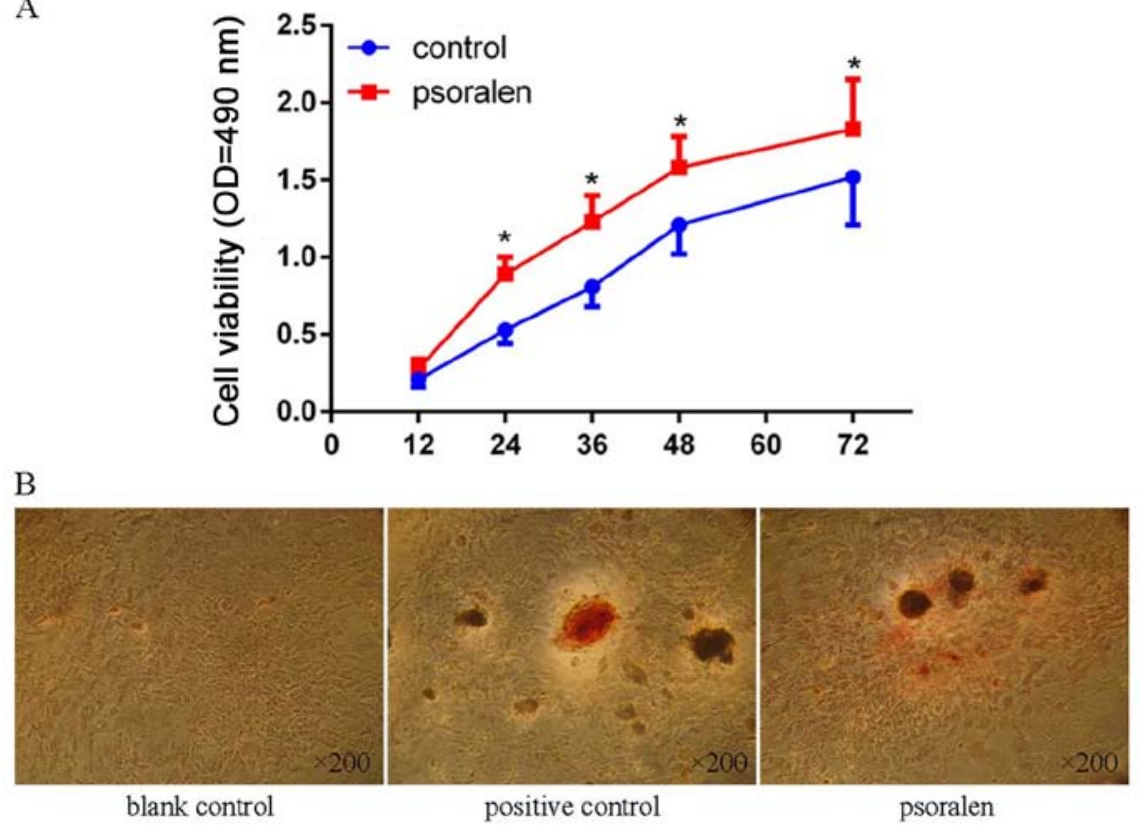

Figure 1. Psoralen promotes the viability and osteogenic differentiation of BMSCs. (A) Cell Counting Kit-8 assay showed that significantly elevated cell viability was found in BMSCs treated with psoralen at all time points except at $12 \mathrm{~h}$. (B) Alizarin red staining (magnification, x200) showed negligible calcium mineral deposition in the blank control group, while similar calcium mineral deposition was observed in the psoralen and positive control groups. " $\mathrm{P}<0.05$ vs. control. BMSCs, bone marrow mesenchymal stem cells; OD, optical density.

the BMSCs were reacted with the appropriate secondary antibody (Bioss, Beijing, PV-0024, PV-0023, 1:100) for $30 \mathrm{~min}$ at $37^{\circ} \mathrm{C}$. The cell nuclei were stained with haematoxylin (Abcam) at room temperature for $1 \mathrm{~min}$ and were visualized by light microscopy (magnification, x200, Leica Microsystems $\mathrm{GmbH})$. The BMSCs were observed at three different views to compare the degree of osteogenic differentiation between the different groups.

Statistical analysis. Each experiment was repeated three times. SPSS 20.0 software (IBM Corp.) was used for the statistical analyses. The data are presented as the mean \pm standard deviation. Student's t-test was used to compare the differences between two groups. One-way ANOVA was used to compare the differences between three or more groups, and Tukey's test was used as a post hoc test to determine which groups were significantly different from each other. $\mathrm{P}<0.05$ was considered to indicate a statistically significant difference. The plots were constructed using GraphPad Prism 6.0 (GraphPad Software, Inc.).

\section{Results}

Psoralen promotes the viability and osteogenic differentiation of BMSCs. The CCK-8 assay showed that cell viability was significantly elevated in BMSCs treated with psoralen at all time points, with the exception of $12 \mathrm{~h}$ (Fig. 1A). The AR-S showed negligible calcium mineral deposition in the blank control group, while similar calcium mineral deposition was observed in the psoralen and positive control groups (Fig. 1B).

miR-488 is downregulated in BMSCs during psoralen-induced osteogenic differentiation. To study the different expression levels of miRNAs during psoralen-induced osteogenic differentiation, BMSCs were treated with psoralen for 3 days and then the cells were subjected to miRNA microarray analysis. In total, 91 miRNAs were differentially expressed between the control and psoralen groups. Compared with the control group, 55 miRNAs were upregulated and 36 were downregulated in the psoralen group. The data were analysed and illustrated in a heat map (Fig. 2A). Among the miRNAs, miR-122, miR-154, miR-488 and miR-205 were randomly selected to confirm the results of the miRNA microarray analysis via RT-qPCR. Compared with the control group, miR-205 was upregulated, while miR-122, miR-154 and miR-488 were downregulated, and miR-488 was the most downregulated (Fig. 2B), which was consistent with the results of the miRNA microarray analysis.

Runx2 is a potential target of $m i R-488$. To gain insight into the molecular mechanisms by which miRNAs regulate the osteogenic differentiation of BMSCs, the potential miRNAs were predicted using TargetScan and it was identified that the osteogenic transcription factor Runx 2 has miR-488 binding sites in its 3'UTR (Fig. 3A). None of the other miRNAs regulate the expression of Runx2, thus miR-488 was further investigated. RT-qPCR showed that miR-488 was significantly upregulated in BMSCs transfected with miR-488 mimics (Fig. 3B), and was downregulated in BMSCs transfected with miR-488 inhibitor (Fig. 3C and D). Runx2 was significantly upregulated in BMSCs transfected with pcDNA 3.1-Runx2 (Fig. 3E). These results indicated that miR-488 mimics, miR-488 inhibitor and pcDNA 3.1-Runx 2 were successfully transfected. To further examine whether miR-488 directly targets Runx2, a luciferase reporter assay was performed and it was demonstrated that the miR-488 mimics significantly inhibited the luciferase activity of the wild-type Runx2 3'UTR but not that of the mutated 

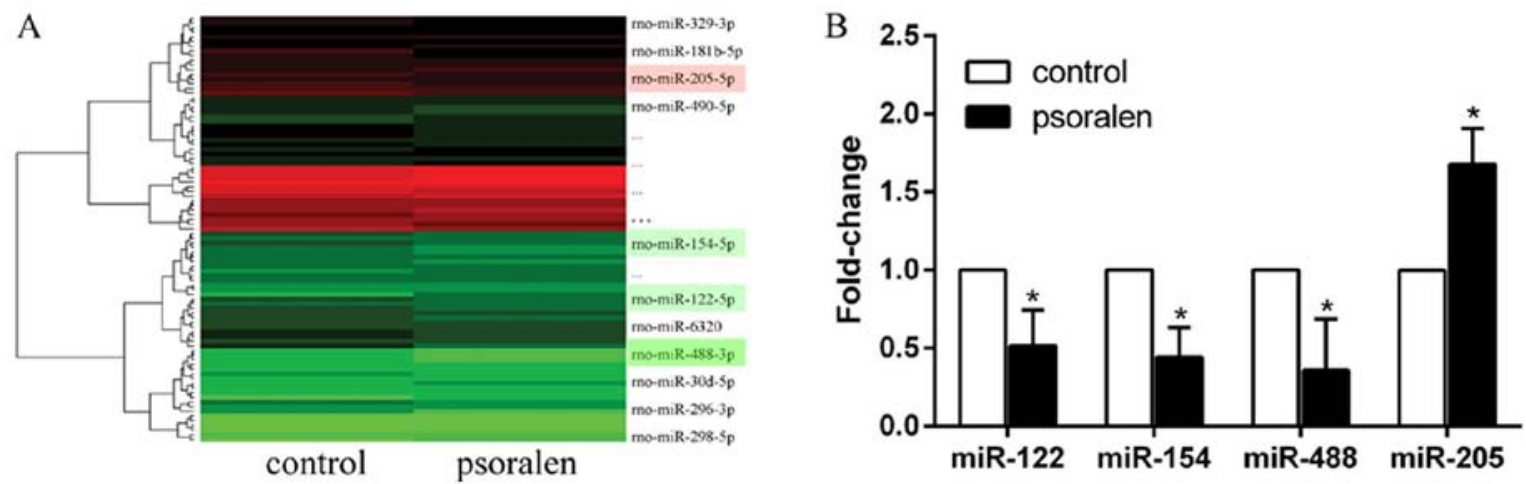

Figure 2. Heat map and bar graph of differentially expressed miRs during the psoralen-induced osteogenic differentiation of bone marrow mesenchymal stem cells. (A) Compared with the control group, 91 miRs were differentially expressed, among which miR-488 was significantly downregulated. Red indicates upregulation and green indicates downregulation. (B) Reverse transcription-quantitative PCR showed that miR-122, miR-154 and miR-488 were downregulated, while miR-205 was upregulated, compared with the control group. " $\mathrm{P}<0.05$ vs. the respective control group. miR, microRNA.

A

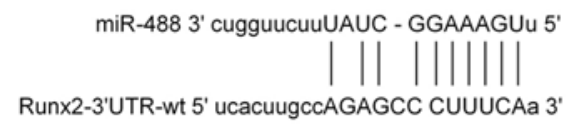

Runx2-3'UTR-mut 5' ucacuugccGCGAGAUGCCAGa 3'

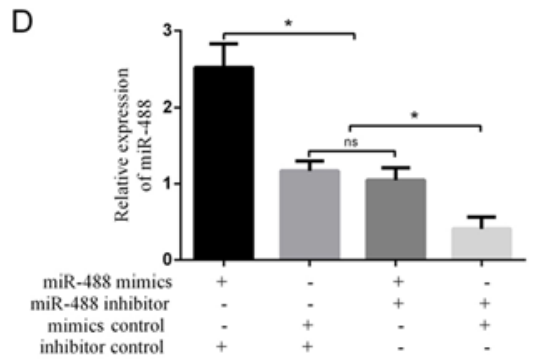

B

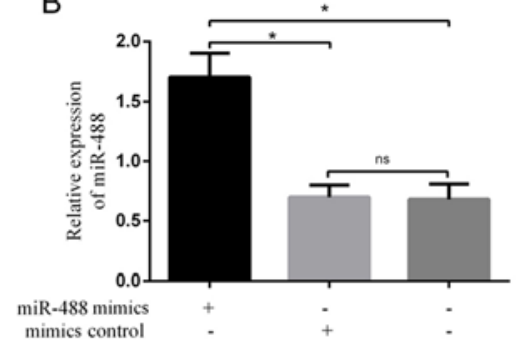

$\mathrm{E}$

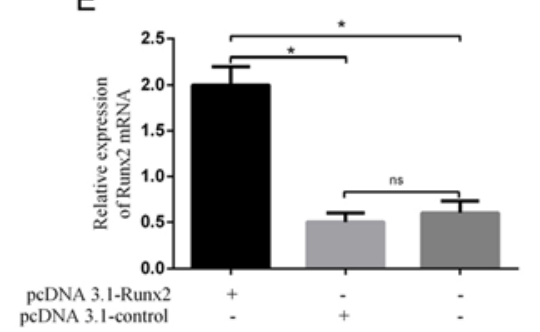

C

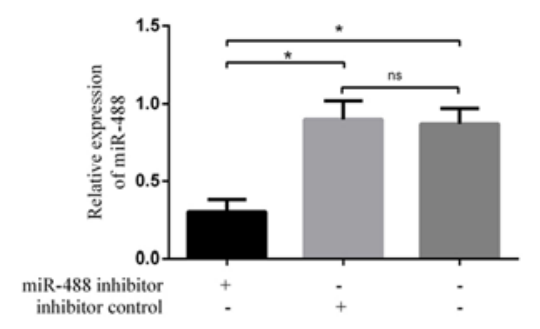

$\mathrm{F}$

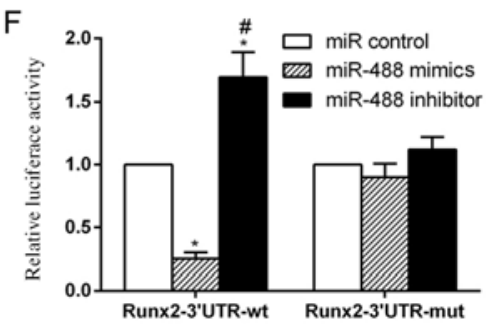

Figure 3. Runx 2 is a potential target of miR-488. (A) Construction profile of the pLUC-Runx 2 vector is presented in the diagram, which contained the miR-488 target sites in Runx2 3'UTR-wt, red sequence presented the mutation region. Reverse transcription-quantitative PCR showed that (B) miR-488 mimics and (C) miR-488 inhibitor were successfully transfected into cells. (D) Reverse transcription-quantitative PCR showed cotransfection of miR-488 mimics and miR-488 inhibitor. Reverse transcription-quantitative PCR showed that (E) pcDNA 3.1-Runx2 was successfully transfected into cells. "P<0.05. (F) Relative luciferase activity demonstrated that Runx 2 is a potential target of miR- 488 . ${ }^{*} \mathrm{P}<0.05$ vs. the miR control group; ${ }^{\prime} \mathrm{P}<0.05$ vs. the miR- 488 mimics group. Runx2, runt-related transcription factor 2; miR, microRNA; 3'UTR, 3'-untranslated region; wt, wild-type; mut, mutant; ns, not significant.

3'UTR (Fig. 3F). Inversely, the miR-488 inhibitor significantly enhanced the luciferase activity of the wild-type Runx2 3'UTR but not that of the mutated 3'UTR (Fig. 3F).

miR-488 negatively regulates the osteogenic differentiation of BMSCs. To better understand the functional role of miR-488 in the osteogenic differentiation of BMSCs, the expressions of Runx2, Osterix and ALP were detected after the BMSCs were transfected with the miR-488 mimics or inhibitor. As shown in Fig. 4A-C, the mRNA and protein expression levels of Runx2, Osterix and ALP were significantly decreased in the miR-488 mimics group but were significantly increased in the miR-488 inhibitor group compared with the control group. The AR-S result also showed that more calcium mineral deposition was found in the miR-488 inhibitor group than in the control and miR-488 mimic groups (Fig. 4D). Immunocytochemistry detected the expression levels of Runx2, Osterix and ALP expressed in BMSCs, where the cytoplasm was stained (Fig. 4E). The expression levels of Runx2, Osterix and ALP in the miR-488 mimic group were notably lower than those in the miR-488 inhibitor group, indicating that miR-488 suppressed osteogenic differentiation of the BMSCs.

Runx 2 overexpression partially rescues the inhibitory effect of miR-488 on osteogenic differentiation. To further explore the underlying mechanism of miR-488/Runx 2 in the osteogenic differentiation of BMSCs, BMSCs were transfected with miR-488 mimics and pcDNA 3.1-Runx2 under treatment with psoralen. As shown in Fig. 5A, AR-S indicated that more calcium mineral deposition was found in the psoralen+pcDNA 3.1-Runx2+miR-488 mimics group than in the psoralen+miR-488 mimics group, while 
A

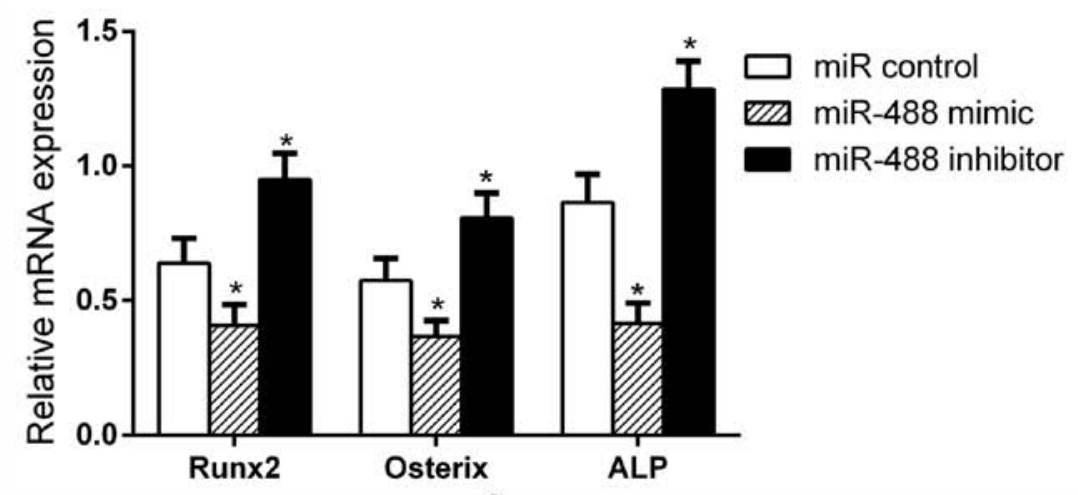

B

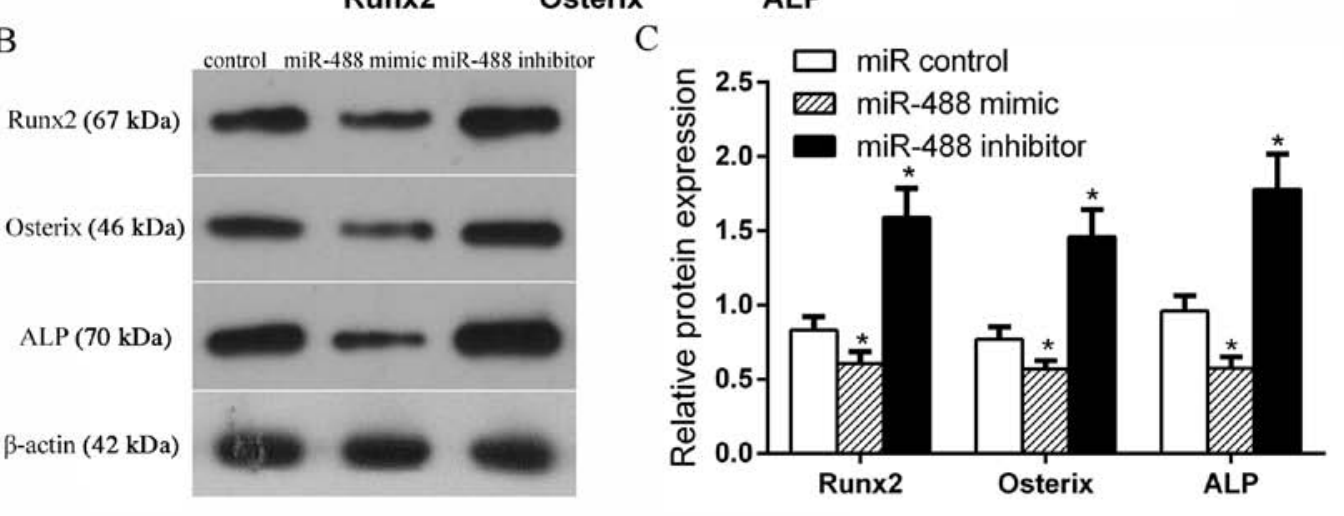

$\mathrm{D}$

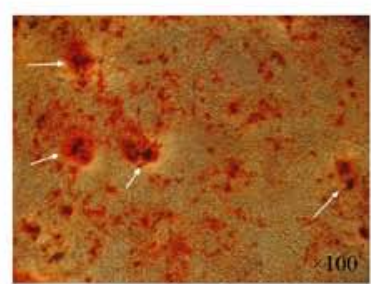

control

E

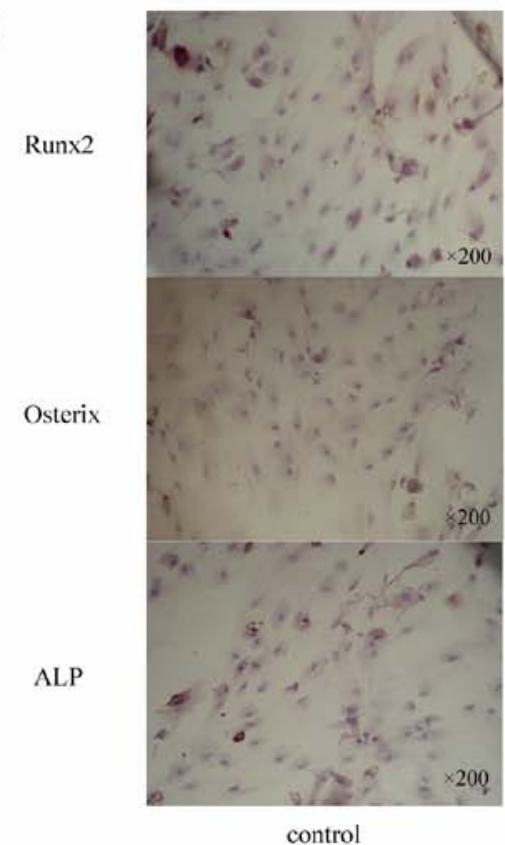

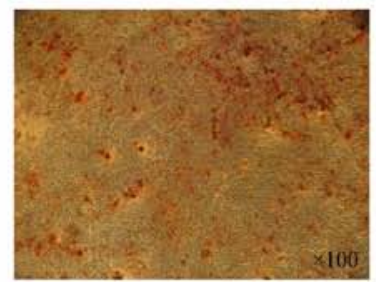

miR-488 mimic

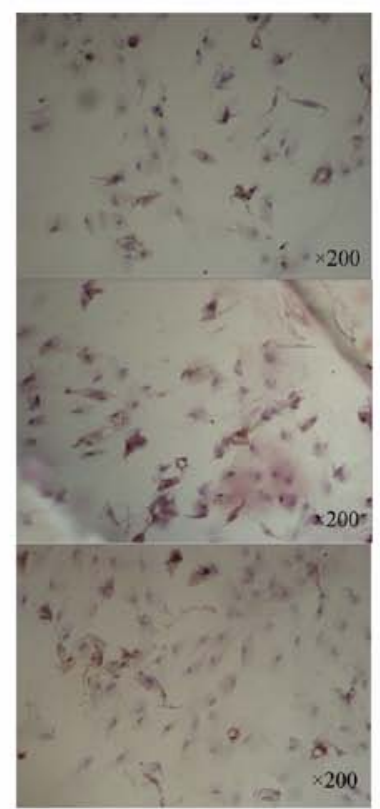

miR-488 mimic

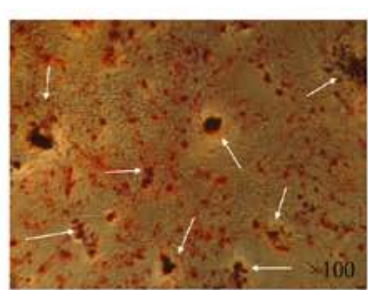

miR-488 inhibitor

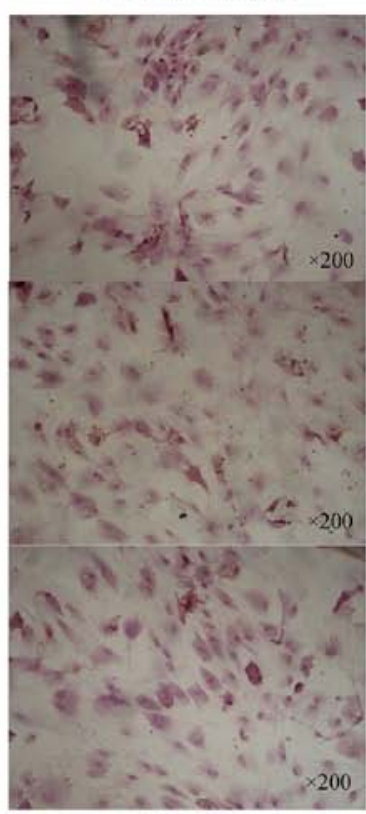

miR-488 inhibitor

Figure 4. miR-488 negatively regulates the osteogenic differentiation of bone marrow mesenchymal stem cells. (A) mRNA expression levels of Runx2, Osterix and ALP were decreased in the miR-488 mimics group and were increased in the miR-488 inhibitor group compared with the control group. Protein expression levels of Runx2, Osterix and ALP were decreased in the miR-488 mimics group and were increased in the miR-488 inhibitor group compared with the control group by (B) western blot analysis and (C) subsequent densitometry. (D) Alizarin red staining (magnification, x100) showed that more calcium mineral deposition was found in the miR-488 inhibitor group than in the control and miR-488 mimics groups. White arrows indicate calcium mineral deposition. (E) Immunocytochemistry (magnification, x200) demonstrated that the expression levels of Runx2, Osterix and ALP in the miR-488 mimics group were notably lower than in the miR-488 inhibitor group. ${ }^{~} \mathrm{P}<0.05$ vs. the respective miR control group. miR, microRNA; Runx2, runt-related transcription factor 2 ; ALP, alkaline phosphatase. 
A

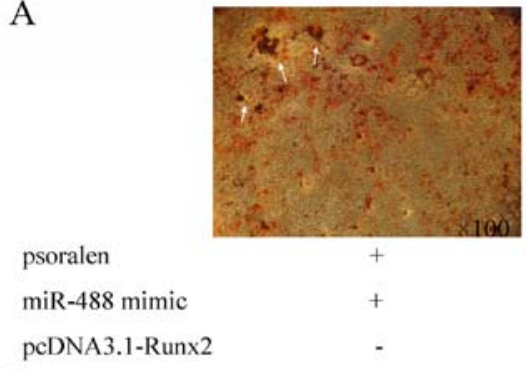

B

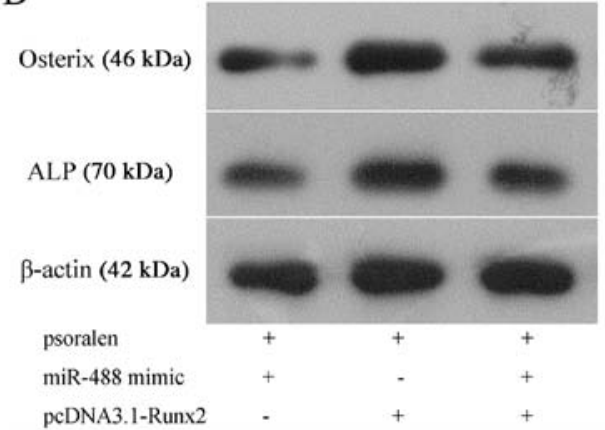

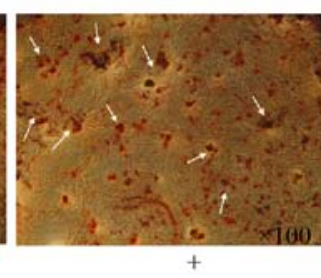

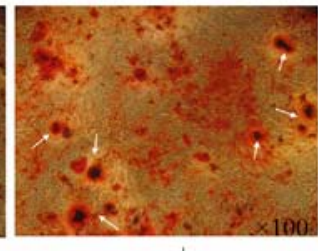

C

$\square$ psoralen+miR-488 mimic

드 ${ }^{2.0}$ ש psoralen+pcDNA 3.1-Runx2

을 psoralen+pcDNA 3.1-Run $\times 2+m i R-488$ mimic

잉 1.5

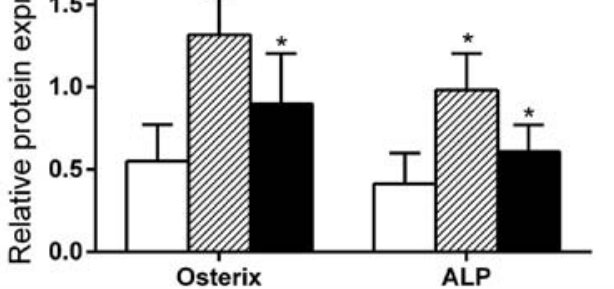

D
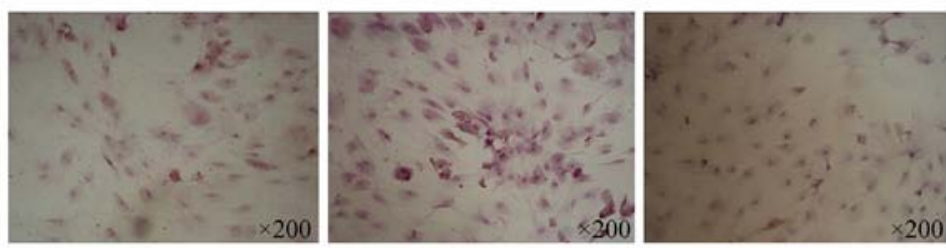

ALP
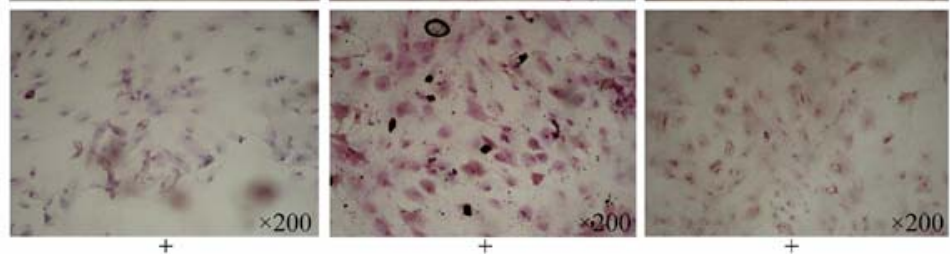

psoralen

miR-488 mimic

pcDNA3.1-Runx2

Figure 5. Runx 2 overexpression partially rescues the inhibitory effect of miR-488 on the osteogenic differentiation of BMSCs. (A) AR-S indicated that more calcium mineral deposition was found in the psoralen+pcDNA 3.1-Runx2+miR-488 mimics group than in the psoralen+miR-488 mimics group, while the calcium mineral deposition in the psoralen+pcDNA 3.1-Runx2+miR-488 mimics group was less than that in the psoralen+pcDNA 3.1-Runx2 group. White arrows indicate calcium mineral deposition. (B) Western blotting and (C) subsequent densitometry, and (D) immunocytochemistry detected the protein expression of osteogenic-specific factors in BMSCs transfected with miR-488 mimics and pcDNA 3.1-Runx2, which was similar to the AR-S results. ${ }^{*} \mathrm{P}<0.05$ vs. the psoralen+miR-488 mimics group. miR, microRNA; BMSCs, bone marrow mesenchymal stem cells; Runx2, runt-related transcription factor 2; AR-S, alizarin red staining.

the calcium mineral deposition in the psoralen+pcDNA 3.1-Runx2+miR-488 mimics group was less than that in the psoralen+pcDNA 3.1-Runx2 group. Runx2 partially rescued the inhibitory effect of miR-488 during the psoralen-induced osteogenic differentiation of BMSCs. Western blotting (Fig. 5B and C) and immunocytochemistry (Fig. 5D) detected the protein expression of osteogenic specific factors in BMSCs transfected with miR-488 mimics and pcDNA 3.1-Runx2, and the results were similar to the AR-S results.

\section{Discussion}

A previous study has shown that psoralen has a smooth muscle diastolic effect, stimulating bone formation and inducing osteogenic differentiation without affecting cell growth (9), consistent with the present findings. However, little is understood regarding the roles of miRNAs in BMSCs induced by psoralen. In the present study, miRNA microarray analysis was conducted to identify the differently expressed miRNAs during psoralen-induced osteogenic differentiation of BMSCs, indicating that miR-488 was significantly decreased. However, the underlying mechanism of miR-488 in regulating BMSCs to stimulate osteogenic differentiation under psoralen induction remains largely unknown.

Each miRNA can bind to hundreds of different mRNAs and assemble with Argonaute proteins into miRNA-induced silencing complexes to direct post-transcriptional silencing of complementary mRNA targets (17). For example, miR-488 has been identified as a cancer-associated miRNA that participates in various diseases, such as hepatocellular carcinoma (18), ovarian cancer (19) and colorectal cancer (20). This is the first time, to the best of the authors' knowledge, that miR-488 has been reported as a key regulator in the osteogenic differentiation of BMSCs. An important mechanism revealed by the 
present study is that Runx 2 is directly targeted by miR-488. Runx2 is a transcription factor that is indispensable for skeletal development and controls bone formation by acting as a signalling hub and transcriptional regulator to coordinate target gene expression $(13,14)$. According to existing literature, miR-690, a Runx2-targeted miRNA, regulated the osteogenic differentiation of $\mathrm{C} 2 \mathrm{C} 12$ myogenic progenitor cells by targeting NF- $\kappa \mathrm{B}$ p65 (21). Heparin-binding EGF-like growth factor and miR-1192 exert opposite effects on Runx2-induced osteogenic differentiation (22). However, to the best of the authors' knowledge, functional evidence of miR-488 targeting Runx 2 in the regulation of BMSCs to undergo osteogenic differentiation has not been previously documented.

Therefore, in the present study, Runx 2 was examined, and the hypothesis that the osteogenic differentiation of BMSCs may be regulated by miR-488 targeting Runx 2 was evaluated. The pLUC-Runx2 plasmid construct, which contained the miR-488 target site in Runx2-3'UTR-wild-type, was constructed to be co-transfected with the miR-488 mimics or inhibitor. The results showed that the miR-488 mimics significantly reduced the luciferase activity of pLUC-Runx 2 , while the miR-488 inhibitor significantly increased the luciferase activity of pLUC-Runx 2 .

To further confirm the hypothesis, the mimics and inhibitor of miR-488 were transfected into BMSCs, and the mRNA and protein expression levels of Runx 2 and a downstream signalling mediator (Osterix) $(14,23)$ and osteogenic-specific marker (ALP) (24) were detected via RT-qPCR, western blotting and immunocytochemistry. The Runx2, Osterix and ALP mRNA and protein expression levels were reduced in the miR-488 mimics group and were enhanced in the miR-488 inhibitor group compared with the control group. The AR-S result also showed that more calcium mineral deposition was found in the miR-488 inhibitor group than the control and miR-488 mimic groups. Further rescue assays demonstrated that Runx 2 overexpression partially rescued the inhibitory effect of miR-488 on osteogenic differentiation under psoralen induction. It should be noted that the present study only observed calcium mineral deposition by AR-S and lacked precise quantification. However, despite this limitation, the present study does support the hypothesis that Runx 2 is targeted by miR-488 and could be directly responsible for the osteogenic differentiation of BMSCs under psoralen induction.

The results of the present study have two important clinical implications. Using the miRNA-target gene network to examine the mechanism of psoralen promoting the osteogenic differentiation of BMSCs, the present study provided insight for the development and application of psoralen in osteoporosis treatment. Additionally, the present study identified a potential molecular approach to promote the osteogenic differentiation of BMSCs, and miR-488/Runx 2 could be therapeutic targets for osteoporosis. miR-488/Runx 2 constitutes a system that has not yet been used in osteoporosis treatment. Inhibition of miR-488 or overexpression of Runx 2 appears to be an interesting novel therapeutic option for osteoporosis.

The present study suggested that miR-488 is involved in the osteogenic differentiation of BMSCs by targeting Runx2 under psoralen induction. Altering miR-488/Runx 2 may be a targeted and mechanism-based therapeutic strategy against osteoporosis.

\section{Acknowledgements}

Not applicable.

\section{Funding}

The present study was supported by the National Natural Science Foundation (grant nos. 81473699 and 81804047), Natural Science Foundation of Guangdong Province (grant nos. 2018A030313694 and 2017A030312009), the Honk Kong Scholar Program (grant no. XJ2018059) and Guangdong Provincial Traditional Chinese Medicine Research Project (grant nos. 20181095 and 20182043).

\section{Availability of data and materials}

The datasets used during the present study are available from the corresponding author on reasonable request.

\section{Authors' contributions}

YH, TJ and YL designed the research. YH, QH, TJ and DC performed the experiments. YH, HS and DC analysed the data. YH and QH wrote the paper. All authors read and approved the final manuscript.

\section{Ethics approval and consent to participate}

All animals received humane care in accordance with the guidelines set by the Care of Experimental Animals Committee of Guangzhou University of Chinese Medicine. Additionally, the present study was approved by the Ethics Committee of Guangzhou University of Chinese Medicine.

\section{Patient consent for publication}

Not applicable.

\section{Competing interests}

The authors declare that they have no competing interests.

\section{References}

1. Collison J: Osteoporosis: Teriparatide preferable for fracture prevention. Nat Rev Rheumatol 14: 4, 2018.

2. Chrischilles EA, Butler CD, Davis CS and Wallace RB: A model of lifetime osteoporosis impact. Arch Intern Med 151: 2026-2032, 1991.

3. Pepe J, Cipriani C, Cecchetti V, et al.: Patients' reasons for adhering to long-term alendronate therapy. Osteoporosis international 2019 May 14. [Epub ahead of print] doi: 10.1007/s00198-019-05010-w.

4. Wang C, Wang J, Li J, Hu G, Shan S, Li Q and Zhang X: KDM5A controls bone morphogenic protein 2-induced osteogenic differentiation of bone mesenchymal stem cells during osteoporosis. Cell Death Dis 7: e2335, 2016.

5. Elango J, Robinson J, Zhang J, Bao B, Ma N, de Val JEMS and Wu W: Collagen peptide upregulates osteoblastogenesis from bone marrow mesenchymal stem cells through MAPK-Runx2. Cells 8, 2019.

6. Qi M, Zhang L, Ma Y, Shuai Y, Li L, Luo K, Liu W and Jin Y Autophagy maintains the function of bone marrow mesenchymal stem cells to prevent estrogen deficiency-induced osteoporosis. Theranostics 7: 4498-4516, 2017.

7. Jing H, Liao L, An Y, Su X, Liu S, Shuai Y, Zhang X and Jin Y: Suppression of EZH2 prevents the shift of osteoporotic MSC fate to adipocyte and enhances bone formation during osteoporosis. Mol Ther 24: 217-229, 2016. 
8. An J, Yang H, Zhang Q, Liu C, Zhao J, Zhang L and Chen B Natural products for treatment of osteoporosis: The effects and mechanisms on promoting osteoblast-mediated bone formation. Life Sci 147: 46-58, 2016

9. Tang DZ, Yang F, Yang Z, Huang J, Shi Q, Chen D and Wang YJ: Psoralen stimulates osteoblast differentiation through activation of BMP signaling. Biochem Biophys Res Commun 405: 256-261, 2011.

10. Czech MP: MicroRNAs as therapeutic targets. N Engl J Med 354: 1194-1195, 2006.

11. Laxman N, Mallmin H, Nilsson O and Kindmark A: miR-203 and miR-320 regulate bone morphogenetic protein-2-induced osteoblast differentiation by targeting distal-less homeobox 5 (Dlx5). Genes (Basel) 8, 2016.

12. Chao C, Li F, Tan Z, Zhang W, Yang Y and Luo C: miR-195 inhibited abnormal activation of osteoblast differentiation in MC3T3-E1 cells via targeting RAF-1. Exp Cell Res 362: 293-301, 2018.

13. Tian Z, Zhou H, Xu Y and Bai J: MicroRNA-495 inhibits new bone regeneration via targeting high mobility group AT-Hook 2 (HMGA2). Med Sci Monit 23: 4689-4698, 2017.

14. Almalki SG and Agrawal DK: Key transcription factors in the differentiation of mesenchymal stem cells. Differentiation 92 : 41-51, 2016.

15. Stephens AS and Morrison NA: Novel target genes of RUNX2 transcription factor and 1,25-dihydroxyvitamin D3. J Cell Biochem 115: 1594-1608, 2014.

16. Livak KJ and Schmittgen TD: Analysis of relative gene expression data using real-time quantitative PCR and the 2(-Delta Delta C(T)) method. Methods 25: 402-408, 2001

17. Meister G: Argonaute proteins: Functional insights and emerging roles. Nat Rev Genet 14: 447-459, 2013.

18. Hu D, Shen D, Zhang M, Jiang N, Sun F, Yuan S and Wan K: MiR-488 suppresses cell proliferation and invasion by targeting ADAM9 and lncRNA HULC in hepatocellular carcinoma. Am J Cancer Res 7: 2070-2080, 2017.
19. Yang Z, Feng Z, Gu J, Li X, Dong Q, Liu K, Li Y and OuYang L: microRNA-488 inhibits chemoresistance of ovarian cancer cells by targeting Six 1 and mitochondrial function. Oncotarget 8: 80981-80993, 2017.

20. Lv Y, Shi Y, Han Q and Dai G: Histone demethylase PHF8 accelerates the progression of colorectal cancer and can be regulated by miR-488 in vitro. Mol Med Rep 16: 4437-4444, 2017.

21. Yu S, Geng Q, Pan Q, Liu Z, Ding S, Xiang Q, Sun F, Wang C, Huang $\mathrm{Y}$ and Hong A: MiR-690, a Runx2-targeted miRNA, regulates osteogenic differentiation of $\mathrm{C} 2 \mathrm{C} 12$ myogenic progenitor cells by targeting NF-kappaB p65. Cell Biosci 6: 10, 2016.

22. Yu S, Geng Q, Ma J, Sun F, Yu Y, Pan Q and Hong A: Heparin-binding EGF-like growth factor and miR-1192 exert opposite effect on Runx2-induced osteogenic differentiation. Cell Death Dis 4: e868, 2013

23. Li Z, Wang W, Xu H, Ning Y, Fang W, Liao W, Zou J, Yang Y and Shao N: Effects of altered CXCL12/CXCR4 axis on $\mathrm{BMP} 2 / \mathrm{Smad} / \mathrm{Runx} 2 / \mathrm{Osterix}$ axis and osteogenic gene expressions during osteogenic differentiation of MSCs. Am J Transl Res 9: 1680-1693, 2017.

24. Kim HK, Cho SG, Kim JH, Doan TK, Hu QS, Ulhaq R, Song EK and Yoon TR: Mevinolin enhances osteogenic genes (ALP, type I collagen and osteocalcin), CD44, CD47 and CD51 expression during osteogenic differentiation. Life Sci 84: 290-295, 2009.

This work is licensed under a Creative Commons Attribution-NonCommercial-NoDerivatives 4.0 International (CC BY-NC-ND 4.0) License. 